\title{
S100A6 and c-Kit-Positive Spindle Cell Melanoma of the Dorsal Foot
}

\author{
Yasutaka Mitamura Takamichi Ito Misa Nakano-Nakamura \\ Hiroshi Uchi Masutaka Furue \\ Department of Dermatology, Graduate School of Medical Sciences, Kyushu University, \\ Fukuoka, Japan
}

\section{Key Words}

Spindle cell melanoma · Differential diagnosis · S100A6 · c-Kit

\begin{abstract}
Spindle cell melanoma, which is a rare form of melanoma, is clinically and histopathologically difficult to diagnose from a variety of nonmelanocytic spindle cell tumors. We describe a 42year-old Japanese woman with amelanotic melanoma that comprised spindle cells with positive c-kit and S100A6 staining. The use of c-kit and S100A6 might be useful for improving the diagnosis.

(c) 2014 S. Karger AG, Basel
\end{abstract}

\section{Introduction}

Spindle cell melanoma (SCM) is a rare form of melanoma that is often amelanotic. Amelanotic melanomas exhibit the following morphologies: epitheloid (72\%), spindle (18.7\%), and desmoplastic (5.3\%) [1]. SCM is clinically and histopathologically difficult to diagnose and can be mistaken for a variety of nonmelanocytic spindle cell tumors. We report the case of an amelanotic melanoma that comprised spindle cells with positive c-kit and S100A6 staining.

\section{Case Presentation}

A 42-year-old Japanese woman was referred to our hospital with a left dorsal foot nodule of 12 months' duration. Physical examination revealed a pedunculated red nodule with a yellow crust that measured $2 \mathrm{~cm}$ in diameter (fig. 1a). The patient's medical history and 
routine laboratory findings were unremarkable. PET-CT showed an area of high uptake in the left inguinal lymph node. We resected the tumor with a 1-cm margin. Histopathologically, the protruding tumorous lesion showed a proliferation of spindle-shaped cells with prominent nucleoli and no pigmentation (fig. 1b, c). Breslow's thickness was $4.9 \mathrm{~mm}$, and the tumor was ulcerated. The cells were positive for microphthalmia-associated transcription factor and c-kit (fig. 2a, b) as well as focally positive for S100 and human melanoma black45, but negative for Melan-A, tyrosinase, and keratin AE1/AE3. Cytoplasmic staining for S100A6 and the receptor for advanced glycation endproducts, a multi-ligand receptor that binds a variety of proteins including S100 family proteins, were observed (fig. 2c). S100A2 and A7 staining were seen in peripheral areas. Staining for other S100 subtypes (S100A4 and S100P) was negative. We diagnosed the tumor as a SCM. Wide resection of the nodule with a 1-cm margin around the tumor and left inguinal dissection was performed. Of the 8 lymph nodes, 2 were metastatic. Twelve months after surgery, no symptoms of recurrence or metastasis were noted.

\section{Discussion}

Desmoplastic melanoma (DM) should be ruled out first before making the diagnosis of SCM. Formally, the diagnostic categories indicate SCM when the collagen content is $<10 \%$, mixed spindle/desmoplastic melanoma when the collagen content is $10-90 \%$, and desmoplastic melanoma when the collagen content is $>90 \%$. The collagen amount is not the only differing factor; patients with DM also have less frequent lymph node involvement and tend to have a less aggressive clinical course [2, 3]. Weissinger et al. [3] reported a diagnostic algorithm for distinguishing DM from SCM. The authors reported that positive c-kit, HMB45 and Melan-A expression were observed significantly more often in SCM, and their data indicated the highest Melan-A sensitivity in SCM. The current case was negative for Melan-A and only focally positive for HMB45, so we used c-kit to distinguish DM from SCM and it was diffusely positive for c-kit. Therefore, the use of c-kit might be more useful for improving the diagnosis.

Other differential diagnoses include various malignant spindle cell neoplasms such as atypical fibroxanthoma, malignant fibrous histiocytoma, leiomyosarcoma, squamous cell carcinoma, dermatofibrosarcoma protuberans, and malignant peripheral nerve sheath tumor (MPNST) [4, 5]. S100 is useful for distinguishing malignant melanoma from other malignant spindle cell neoplasms. However, MPNST is usually positive for S100, so it is difficult to distinguish MPNST from SCM. The S100 proteins comprise a family of acidic, lowmolecular weight calcium-binding proteins that regulate various cellular processes via calcium-dependent interactions with target proteins. To date, more than 20 members of this family have been identified, including S100A6. The function of S100A6 has not yet been fully defined, but this protein might play a role in cell proliferation, cytoskeletal dynamics, and tumorigenesis $[6,7]$. Melanomas typically express S100A6 in a weak and patchy pattern $[8$, 9]. Fullen et al. [4] reported that various malignant spindle cell tumors involving the skin or soft tissues were evaluated for S100A6 expression. In that study, S100A6 staining of spindled tumor cells was detected in 32 out of 33 (97\%) evaluated DM lesions. In contrast, MPNST lesions failed to express S100A6 or showed a weak expression. To our knowledge, there have been no reported cases of SCM with positive S100A6 staining. Thus, S100A6 might provide a clue when distinguishing MPNST from SCM. 
Mitamura et al.: S100A6 and c-Kit-Positive Spindle Cell Melanoma of the Dorsal Foot

\section{Disclosure Statement}

The authors declare no conflict of interest.

\section{References}

$\checkmark 1$ Cheung WL, Patel PR, Leonard A, Firoz B, Meehan SA: Amelanotic melanoma: a detailed morphologic analysis with clinicopathologic correlation of 75 cases. J Cutan Pathol 2012;39:33-39.

$\checkmark 2$ Chen LL, Jaimes N, Barker CA, Busam KJ, Marghoob AA: Desmoplastic melanoma: a review. J Am Acad Dermatol 2013;149:413-421.

-3 Weissinger SE, Keil P, Silvers DN, et al: A diagnostic algorithm to distinguish desmoplastic from spindle cell melanoma. Mod Pathol 2014;27:524-534.

4 Fullen DR, Garrisi AJ, Sanders D, Thomas D: Expression of S100A6 protein in a broad spectrum of cutaneous tumors using tissue microarrays. J Cutan Pathol 2008;35:28-34.

5 Sakamoto A: Atypical fibroxanthoma. Clin Med Oncol 2008;2:117-127.

-6 Leśniak W, Słomnicki ŁP, Filipek A: S100A6 - new facts and features. Biochem Biophys Res Commun 2009;390:1087-1092.

7 Ribé A, McNutt NS: S100A6 protein expression is different in Spitz nevi and melanomas. Mod Pathol 2003;16:505-511.

-8 Zhu L, Kohda F, Nakahara T, et al: Aberrant expression of S100A6 and matric metalloproteinase 9, but not S100A2, S100A4, and S100A7, is associated with epidermal carcinogenesis. J Dermatol Sci 2013;72:311319.

-9 Puri PK, Elston CA, Tyler WB, Ferringer TC, Elston DM: The staining pattern of pigmented spindle cell nevi with S100A6 protein. J Cutan Pathol 2011;38:14-17. 
Mitamura et al.: S100A6 and c-Kit-Positive Spindle Cell Melanoma of the Dorsal Foot
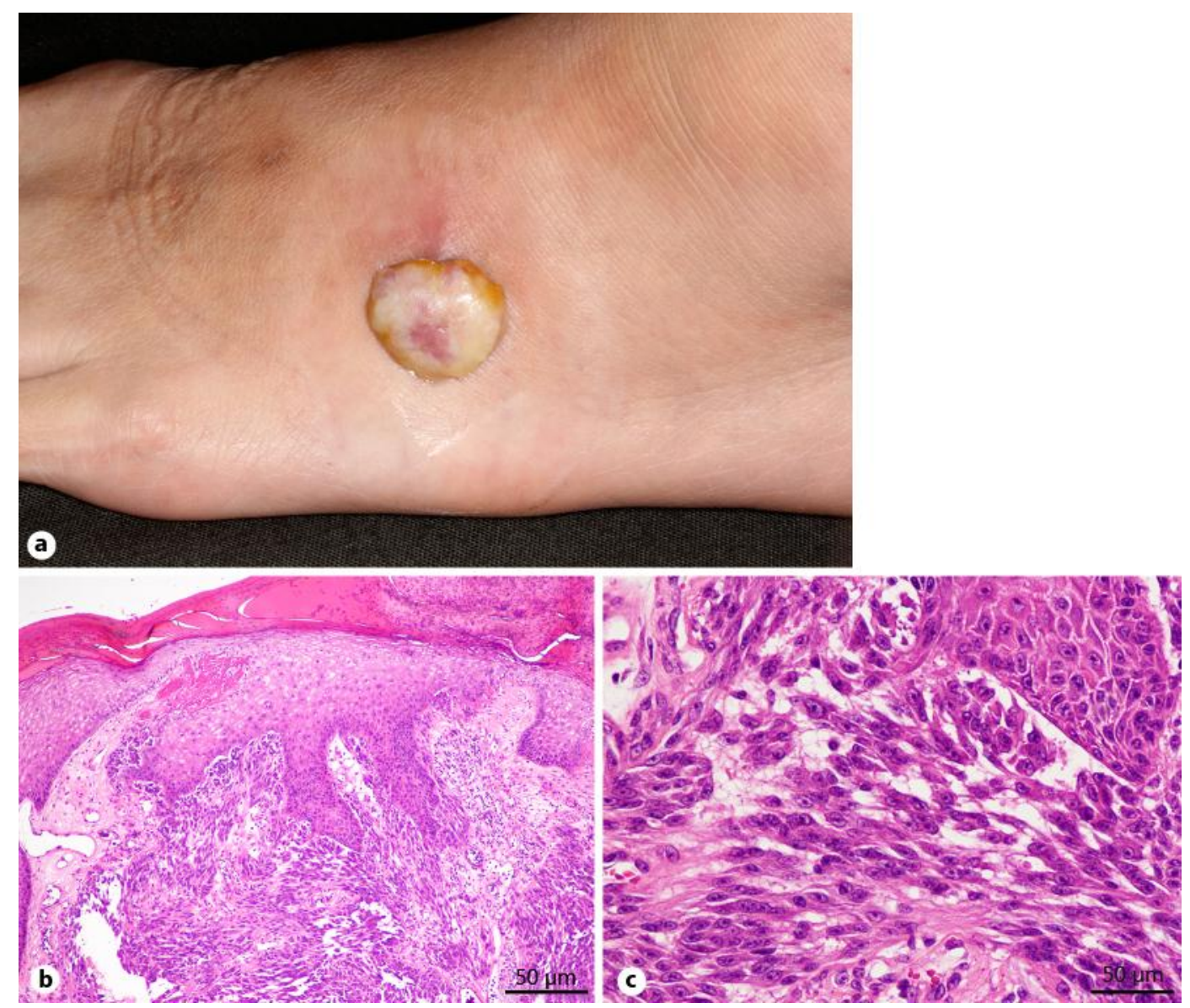

Fig. 1. a A pedunculated red nodule with a yellow crust on the left dorsal foot. b Invasion of tumor cells from the dermal-epidermal junction into the dermis. The tumor comprised spindle cells with a low collagen content $(<10 \%)$ (HE staining. $\times 40$ ). c Higher-power magnification of the tumor at the dermalepidermal junction. The tumorous lesion showed a proliferation of spindle-shaped cells with prominent nucleoli and some mitosis (HE staining. $\times 200$ ). 

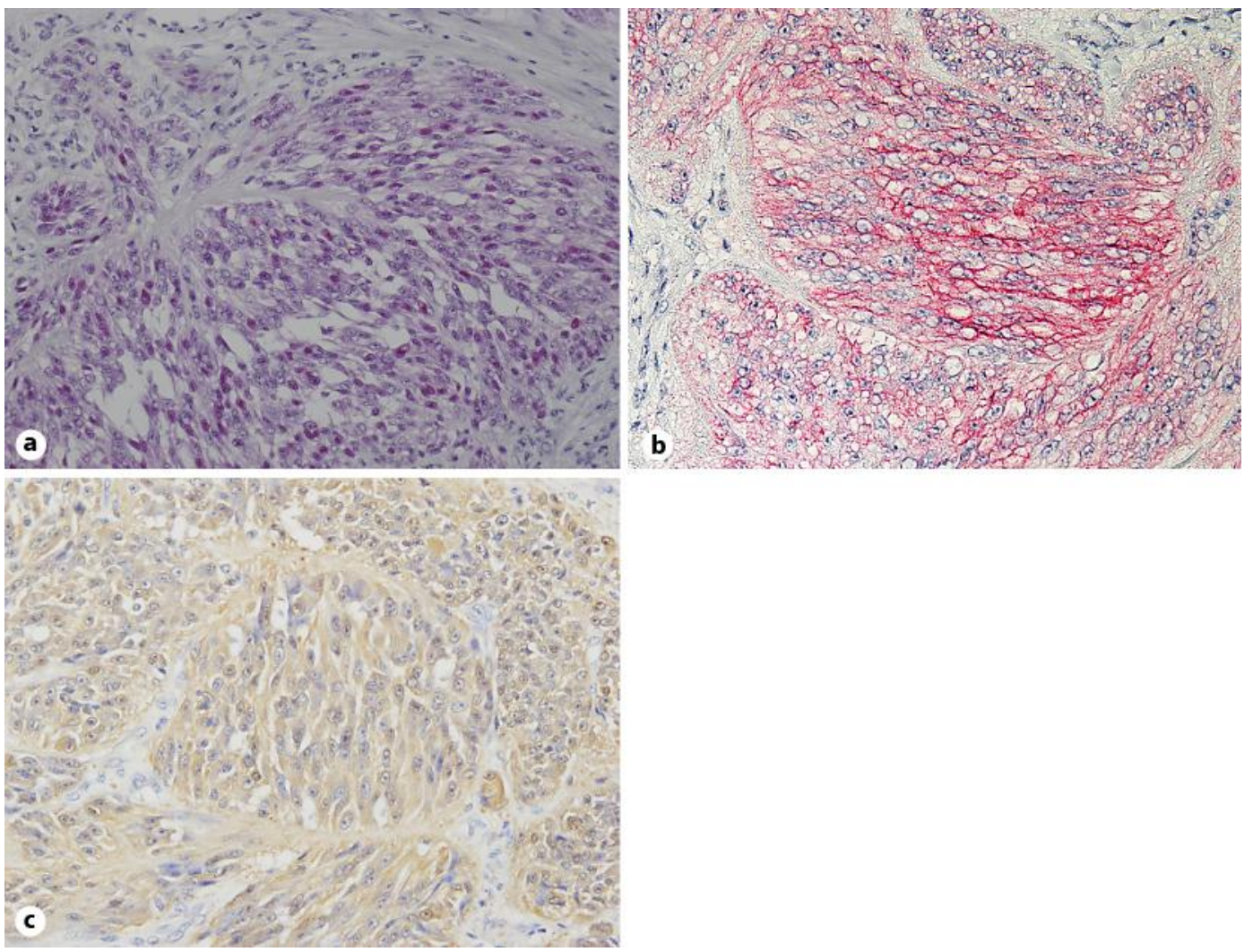

(C) 2014 S. Karger AG, Basel

www.karger.com/cde

Mitamura et al.: S100A6 and c-Kit-Positive Spindle Cell Melanoma of the Dorsal Foot

Fig. 2. a The microphthalmia-associated transcription factor stain showed positive nuclear staining $(\times 400)$. b The c-kit stain showed positive membranous staining $(\times 400)$. c The S100A6 stain showed positive cytoplasmic staining $(\times 400)$. 